\title{
GENERALIZED FRACTIONAL PROGRAMMING DUALITY: A RATIO GAME APPROACH
}

\author{
S. CHANDRA ${ }^{1}$, B. D. CRAVEN ${ }^{2}$, AND B. MOND ${ }^{3}$
}

(Received 8 May 1985; revised 31 October 1985)

\begin{abstract}
A ratio game approach to the generalized fractional programming problem is presented and duality relations established. This approach suggests certain solution procedures for solving fractional programs involving several ratios in the objective function.
\end{abstract}

\section{Introduction}

Duality in fractional programming has been studied extensively (see for example [14], [15]) but mostly it has been confined to the optimization of a single ratio. Recently, some duality results have been obtained for fractional programs involving several ratios, notably by Crouzeix [3], Crouzeix, Ferland and Schaible [4] and Jagannathan and Schaible [10]. The basic approach in [3] and [4] has been to use the quasiconvex duality theory of [3], while in [10] duality results were obtained using Farkas's Lemma. Many applications of duality of generalized fractional programming have been given in [4], which also includes results like the complementarity slackness theorem for generalized linear fractional programming.

In this paper, we study duality for such nonconvex programs by a different approach, based on ratio games [16]. The use of ratio games in generalized fractional programming is very natural, since both of them have their origin in von Neumann's model [19] of an expanding economy. This approach is not only

\footnotetext{
${ }^{1}$ Mathematics Department, Indian Institute of Technology, Hauz Khas, New Delhi-110016, India, and Mathematics Department, University of Melbourne, Parkville, Victoria 3052, Australia

${ }^{2}$ Mathematics Department, University of Melbourne, Parkville, Victoria 3052, Australia

${ }^{3}$ Department of Mathematics, La Trobe University, Bundoora, Victoria 3083, Australia

(c) Copyright Australian Mathematical Society 1986, Serial-fee code 0334-2700/86
} 
simple but also promising from an algorithmic point of view. We treat the linear case of such problems in section 2, while the nonlinear (convex) case is discussed in section 3. Some remarks about algorithmic aspects of such problems are included in section 4 .

\section{The linear case}

Consider the generalized linear fractional programming problem:

(P):

$$
\operatorname{Min}_{x \in X} \operatorname{Max}_{1 \leqslant t \leqslant p}\left\{\left[\left(\sum_{j=1}^{n} a_{i j} x_{j}\right)+\alpha_{i}\right] /\left[\left(\sum_{j=1}^{n} b_{i j} x_{\jmath}\right)+\beta_{i}\right]\right\},
$$

where $X=\left\{x \in R^{n}: x \geqslant 0, C x \leqslant \gamma\right\}, C$ being an $(m \times n)$ matrix and $\gamma$ an $m$-dimensional vector. Also $A=\left[a_{i j}\right]$ and $B=\left[b_{i j}\right]$ are $(p \times n)$ matrices, $\alpha=$ $\left(\alpha_{1}, \alpha_{2}, \ldots, \alpha_{p}\right)^{T}$ and $\beta=\left(\beta_{1}, \beta_{2}, \ldots, \beta_{p}\right)^{T}$ are vectors in $R^{p}$. The symbol $\langle x, y\rangle$ denotes the scalar product of vectors $x, y \in R^{n}$.

We shall assume the following for program $(\mathrm{P})$ :

(H1) $X$ is nonempty.

(H2) $(B x+\beta)>0$ for all $x \in X$.

(H3) $X$ is compact.

Assumptions (H1) and (H2) are the same as those in [4] and [10]. The additional assumption $(\mathrm{H} 3)$ is made to facilitate the use of ratio games. Also this assumption will be relevant if we wish to study the algorithmic aspects of the generalized fractional program (P).

\subsection{A ratio game framework: motivation and formulation}

For all $x \in X$, let $\rho(x)$ be defined by

$$
\rho(x)=\operatorname{Max}_{1 \leqslant i \leqslant p}\left\{\left[\left(\sum_{j=1}^{n} a_{i j} x_{j}\right)+\alpha_{i}\right] /\left[\left(\sum_{j=1}^{n} b_{i j} x_{j}\right)+\beta_{i}\right] .\right.
$$

Then program (P) can equivalently be expressed as the following program (EP) for the variables $x$ and $\lambda$ :

(EP): Minimize $\lambda$ subject to

$$
\left[\left(\sum_{j=1}^{n} a_{i j} x_{j}\right)+\alpha_{i}\right] /\left[\left(\sum_{j=1}^{n} b_{i j} x_{j}\right)+\beta_{i}\right] \leqslant \lambda, \quad x \in X .
$$

The program (EP) is, in general, nonconvex.

Let us now take a special case of $(\mathrm{P})$ which motivates a game-theoretic approach. Let $\alpha_{i}=0, \beta_{i}=1$ for all $i$ and $b_{i j}=0$ for all $i$ and $j$. Program (P) 
then reduces to a generalized linear program, namely

$$
\operatorname{Min}_{x \in X} \underset{1 \leqslant i \leqslant p}{\operatorname{Max}}\left(\sum_{j=1}^{n} a_{i j} x_{\jmath}\right),
$$

and (EP) becomes a linear program. Now, by taking

$$
X=\left\{x \in R^{n}: x \geqslant 0, \sum_{j=1}^{n} x_{j}=1\right\},
$$

we observe that (EP) becomes the linear program for the minimizing player in the matrix game $y^{T} A x, x \in X, y \in Y$, where $Y=\left\{y \in R^{p}: y \geqslant 0, \sum_{i=1}^{p} y_{i}=1\right\}$. Here $Y$ denotes the set of mixed strategies for Player I, and $X$ denotes the set of mixed strategies for Player II. It is simple to observe that this analogy between generalized linear programming and matrix game theory remains valid even for the case when $X$ is a compact convex subset of $R^{n}$, and $\alpha_{1}$ are not necessarily zero for all $i$. Thus, solving a generalized linear program can be considered as solving a matrix game for Player II where the minimizing player, Player II, chooses his strategies $x$ from a compact convex subset $X \subseteq R^{n}$, and the maximizing player, Player I, chooses his strategies from the set of mixed strategies $Y=\left\{y \in R^{p}: y \geqslant 0, \sum_{t=1}^{p} y_{t}=1\right\}$. Such a game will have a solution, in view of the general minimax theorems (Fan [7], Karlin [11]).

We now wish to show that an analogy, similar to the above, exists between generalized linear fractional programming and (zero-sum) ratio game theory. For this, we take $Y$ as before, and consider the ratio game $K(y, x)=P(y, x) / Q(y, x)$ where

$$
\begin{aligned}
& P(y, x)=y^{T} A x+y^{T} \alpha, \\
& Q(y, x)=y^{T} B x+y^{T} \beta .
\end{aligned}
$$

Note that, in view of (H2), $Q(y, x)>0$ for all $y \in Y$ and $x \in X$. Also, this ratio game will have a solution (see von Neumann [19], Lommis [12] and Shapley [17]). Now we have the following Lemmas:

Lemma 1. Let $x \in X$ be arbitrary but fixed; let $K(y, x)=P(y, x) / Q(y, x)$. Then $\operatorname{Max}_{y \in Y} K(y, x)=\rho(x)$.

Proof. For $v \geqslant \rho(x), x \in X$, and $y \in Y$,

$$
\begin{aligned}
P(y, x)-v Q(y, x) & \leqslant \operatorname{Max}_{1 \leqslant i \leqslant p}\left\{\left(\sum_{j=1}^{n} a_{i j} x_{\jmath}\right)+\alpha_{\imath}-v\left[\left(\sum_{j=1}^{n} b_{\imath} x_{j}\right)+\beta_{\imath}\right]\right\} \\
& =0 \text { when } v=\rho(x) .
\end{aligned}
$$

The result follows since $Q(y, x)>0$. 
Lemma 2. (Schroeder [16]). A necessary and sufficient condition for $\hat{v}$ to be the value of the ratio game $K(y, x)$ and $\hat{y}, \hat{x}$ optimal strategies is that the two person, zero sum game with payoff function $P(y, x)-\hat{v} Q(y, x)$ has value zero and optimal strategies $\hat{y}, \hat{x}$.

Here, it is to be noted that the ratio game $K(y, x)$ is slightly more general than Schroeder's ratio game [16] but essentially the same proof holds for Lemma 2.

Lemma 3. Let $\bar{x}$ be an optimal solution of (P) with the optimal value $\bar{v}$. Then $\bar{v}$ is the value of the ratio game $K(y, x)$ and $\bar{x}$ is an optimal strategy for Player II and conversely.

Proof. The proof follows because of Lemma 1 and the equivalence between (P) and (EP).

Lemma 2 is related to the parametric optimization method of [6] and [8]. However, in [6] and [8] there is only one ratio in the objective function. As is shown here, the ratio game treated in [16] is equivalent to the generalized fractional programming problem of [3], [4] and [10], namely (P); see also [1] and [5]. Lemmas 1 and 3 also follow from

$$
\operatorname{Max}_{y \in Y} x^{T} A y / x^{T} B y=\operatorname{Max}_{1 \leqslant 1 \leqslant p}\left[\sum_{j} a_{i j} x_{j} / \sum b_{i j} x_{j}\right],
$$

since the maximum of a quasiconvex ratio is reached at an extreme point.

In view of the above Lemmas, solving the generalized fractional program $(P)$ is equivalent to solving the ratio game $K(y, x)$ for Player II and this can be achieved in a sequential fashion by solving the game $P(y, x)-v Q(y, x)$. We shall exploit these Lemmas to study the duality and algorithmic aspects of the generalized fractional program (P).

\subsection{Construction of dual}

For $v \in R$, consider the game $P(y, x)-v Q(y, x)$. If we want to solve the game for Player II then the following linear programming problem is to be solved:

$(\mathrm{LP})_{v}: \quad$ Minimize $q$

subject to

$\sum_{j=1}^{n}\left(a_{i j}-v b_{i j}\right) x_{j}+\left(\alpha_{\imath}-v \beta_{i}\right) \leqslant q, \quad(i=1,2, \ldots, p)$, 


$$
\begin{gathered}
C x \leqslant \gamma, \\
x \geqslant 0 .
\end{gathered}
$$

The optimal solution of $(L P)_{v}$ will give the value of the game $P(y, x)-v Q(y, x)$ and the optimal strategy for Player II with regard to this game. As soon as we are able to choose that $v$ (say $\bar{v}$ ) for which (LP) $)_{\bar{v}}$ has the minimum value zero, both the generalized fractional program (P) and the ratio game $K(y, x)$ for Player II are solved.

Let us now write the dual $D(L P)_{v}$ of $(L P)_{v}$ :

$$
D(L P)_{v}: \text { Maximize }\langle u, \alpha\rangle-v\langle u, \beta\rangle-\langle w, \gamma\rangle
$$

subject to

$$
\begin{gathered}
\left\langle u, a_{. j}\right\rangle-v\left\langle u, b_{. j}\right\rangle+\left\langle w, c_{\cdot j}\right\rangle \geqslant 0,(j=1,2, \ldots, n) \\
\langle u, e\rangle=1, \\
u \geqslant 0, \\
w \geqslant 0 .
\end{gathered}
$$

Here $a_{. j}, b_{. j}$ and $c_{. j}(j=1,2, \ldots, n)$ denote the $j$ th column of $A, B$ and $C$ respectively, $e^{T}=(1,1, \ldots, 1)$ and $u \in R^{p}, w \in R^{m}$ are dual variables. Also, in view of (9) and (10), $u \neq 0$.

For $v=\bar{v}$, the minimum value of $(L P)_{\bar{v}}$ is zero and so there exist $\bar{u}, \bar{w}$ optimal to $D(L P)_{\bar{v}}$ such that

$$
\langle\bar{u}, \alpha\rangle-\bar{v}\langle\bar{u}, \beta\rangle-\langle\bar{w}, \gamma\rangle=0 .
$$

Further, assuming that $\beta \geqslant 0$ and $B \geqslant 0$ (these assumptions are made temporarily to get some motivation for the dual $(D)$ of $(P)$ and will be dropped later for proving the duality results) we observe that for any $v \leqslant \bar{v}$, these $\bar{u}$ and $\bar{w}$ will remain feasible to $D(L P)_{v}$ and

$$
[\langle\bar{u}, \alpha\rangle-v\langle\bar{u}, \beta\rangle-\langle\bar{w}, \gamma\rangle] \geqslant 0 .
$$

Therefore the desired $\bar{v}$ is the supremum of those $v$ for which (12) holds. This suggests the following dual $(D)$ to the generalized fractional program $(P)$ :

Dual $(D)$ :

Maximize $v$ subject to

$$
\begin{aligned}
& \langle u, \alpha\rangle-v\langle u, \beta\rangle-\langle w, \gamma\rangle \geqslant 0, \\
& \left\langle u, a_{. j}\right\rangle-v\left\langle u, b_{. j}\right\rangle+\langle w, c .,\rangle \geqslant 0, \quad(j=1,2, \ldots, n) \\
& u \geqslant 0, w \geqslant 0, \\
& u \neq 0 \text {, } \\
& v \in R, u \in R^{p}, w \in R^{m} .
\end{aligned}
$$

We prove the basic duality results between primal $(P)$ and dual $(D)$ in the next section. 


\subsection{Duality}

Theorem 1 (Weak Duality Theorem). Let $(x, \lambda)$ be feasible to $(P)$ and $(u, v, w)$ be feasible to $(D)$. Then

$$
v \leqslant \lambda .
$$

Further, if equality holds at $(18)$ then $(x, \lambda)$ and $(u, v, w)$ are optimal for the respective problems.

Proof. Multiplying (14) by $x_{j} \geqslant 0$ and summing over $j$, then adding to (13), we get

$$
\sum_{i=1}^{p} u_{i}\left[\sum_{j=1}^{n}\left(a_{i j}-v b_{i j}\right) x_{J}+\left(\alpha_{\imath}-v \beta_{\imath}\right)\right]+\sum_{k=1}^{m} w_{k}\left[\sum_{j=1}^{n} c_{k J} x_{\jmath}-\gamma_{k}\right] \geqslant 0 .
$$

Now, if possible, let $\lambda<v$. Then because of the constraints of $(E P)$,

$$
\left[\sum_{\jmath=1}^{n}\left(a_{\imath \jmath}-v b_{\imath \jmath}\right) x_{\jmath}+\left(\alpha_{\imath}-v \beta_{\imath}\right)\right]<0 \text { for all } i=1,2, \ldots, p \text {. }
$$

Also $u \neq 0$ by (16) and hence the first term on the left hand side of (19) is strictly negative $(<0)$. Further $w \geqslant 0$ and $C x \leqslant \gamma$ and hence the second term on the left hand side of $(19)$ is nonpositive $(\leqslant 0)$. Thus the left hand side of $(19)$ is strictly negative $(<0)$ and hence a contradiction to (19). Therefore $\lambda \geqslant v$.

The other part of the theorem is immediate.

TheOREM 2 (Duality Theorem). Let $\bar{x}, \bar{v}$ (respectively) be the optimal solution and the optimal value of the generalized fractional program $(P)$. Then there exist $\bar{u}$ and $\bar{w}$ such that $(\bar{u}, \bar{v}, \bar{w})$ is optimal to $(D)$ with $\bar{v}$ as its optimal value.

Conversely, let $(\bar{u}, \bar{v}, \bar{w})$ be optimal to $(D)$ with optimal value $\bar{v}$, then there exists $\bar{x} \in X$ such that $\bar{x}$ is optimal to $(P)$ with the optimal value $\bar{v}$.

Proof. $\bar{x}, \bar{v}$ (respectively) are the optimal solution and the optimal value of the generalized fractional program $(P)$.

$\Rightarrow \bar{v}$ is the value of the ratio game $K(y, x)$ and $\bar{x}$ is an optimal strategy for Player II.

$\Rightarrow$ The matrix game $P(y, x)-\bar{v} Q(y, x)$ has the value zero and $\bar{x}$ is an optimal strategy for Player II with regard to this game (by Lemma 2 ).

$\Rightarrow(L P)_{\bar{v}}$ has optimal solution at $(\bar{x}, 0)$ with minimum value zero.

$\Rightarrow$ There exist $(\bar{u}, \bar{w})$ feasible to $D(L P)_{\bar{v}}$ such that $\langle\bar{u}, \alpha\rangle-\bar{v}\langle\bar{u}, \beta\rangle-\langle\bar{w}, \gamma\rangle=0$.

$\Rightarrow(\bar{u}, \bar{v}, \bar{w})$ is optimal to $(D)$ with the optimal value $\bar{v}$ (by Theorem 1). 
Conversely let $(\bar{u}, \bar{v}, \bar{w})$ be optimal for $(D)$ with the optimal value $\bar{v}$. We want to show that there exists $\bar{x} \in X$ such that $\bar{x}$ is optimal to $(P)$ with the optimal value $\bar{v}$ (i.e. $(\bar{x}, \bar{v})$ is optimal to $(E P)$ with the optimal value $\bar{v}$ ). Note that the existence of $\left(x^{*}, v^{*}\right)$, optimal to $(E P)$, is guaranteed by the minimax theorem of ratio games (von Neumann [19], Lommis [12] and Shapley [17]). Therefore it suffices to prove that $v^{*}=\bar{v}$, because then $\bar{x}$ will be the optimal solution of $(L P)_{\hat{v}}$. Now by the first part of the theorem, the optimality of $\left(x^{*}, v^{*}\right)$ to $(E P)$ implies the existence of $u^{*}$ and $w^{*}$ such that $\left(u^{*}, v^{*}, w^{*}\right)$ is optimal to $(D)$ with the optimal value $v^{*}$. But then, $\bar{v}$ and $v^{*}$ both become optimal values of $(D)$ and hence $v^{*}=\bar{v}$.

\subsection{Remarks}

REMARK 1. It is interesting to observe that the linear programs $(L P)_{v}$ and $D(L P)_{v}$ also occur in [4] but for a different purpose. The basic dual in [4] has been obtained by using the quasiconvex duality of [3] and these linear programs are used to express this basic dual in a more useful form. In our work, the programs $(L P)_{v}$ and $D(L P)_{v}$ occur very naturally by the ratio game theory and we construct the dual $(D)$ by using these programs.

Remark 2. The motivation for writing the dual $(D)$ from $D(L P)_{v}$ is exactly the same as in the fractional programming duality [9] where results of Dinkelbach [6] or Jagannathan [8] are used to write the fractional dual.

Remark 3. The dual and duality relations are established here using linear programming duality and the minimax theorem in game theory. The same dual was obtained in [10] using Farkas's Lemma, which is essentially equivalent to linear programming duality. The present approach, from the ratio game, motivates the form of the dual.

\section{The nonlinear case}

Consider the following generalized nonlinear fractional program: $(N P)$

$$
\operatorname{Min}_{x \in X} \operatorname{Max}_{1 \leqslant 1 \leqslant p}\left[f_{i}(x) / g_{i}(x)\right]
$$

where $X=\left\{x: h_{k}(x) \leqslant 0, k=1,2, \ldots, r\right\}$ and $f_{i}, g_{\imath}$ and $h_{k}$ are real valued continuous functions.

We shall assume the following for program $(N P)$ :

(A1) $f_{l}, h_{k}$ and $-g_{l}$ are continuous convex functions for each $i$ and $k$.

(A2) $g_{i}(x)>0$ for all $i$ and for all $x \in X$. 
(A3) If $g_{1}$ is not affine then $f_{i}(x) \geqslant 0$ for all $x \in X$.

(A4) $X$ is nonempty and compact.

Now as in the linear case, we consider the nonlinear ratio game $J(y, x)=$ $[F(y, x) / G(y, x)], x \in X, y \in Y$, where

$$
F(y, x)=\sum_{\imath=1}^{p} y_{\imath} f_{t}(x) ; G(y, x)=\sum_{i=1}^{p} y_{l} g_{l}(x)
$$

and

$$
Y=\left\{y: y \geqslant 0, \sum_{t=1}^{p} y_{t}=1\right\} .
$$

The following results are true for the ratio game $J(y, x)$ :

THEOREM 3. Under the assumptions (A1) to (A4)

$$
\operatorname{Min}_{x \in X} \operatorname{Max}_{y \in Y} J(y, x)=\underset{y \in Y}{\operatorname{Max}} \operatorname{Min}_{x \in X} J(y, x) .
$$

Proof. The proof follows from Sion's Theorem [18] by observing that $X$ and $Y$ are compact convex sets and under the assumptions (A1) to (A4), $J(y, \cdot)$ is pseudoconvex and $J(\cdot, x)$ is pseudoconcave. Alternatively, the proof based on a fixed point theorem (Karlin [11]) can be modified for this special case of fractional functions.

Theorem 4. A necessary and sufficient condition for $\hat{v}$ to be the value of the nonlinear ratio game $J(y, x)$ with $\hat{y}, \hat{x}$ as optimal strategies is that the two person zero sum game $F(y, x)-\hat{v} G(y, x)$ has value zero and optimal strategies $\hat{y}, \hat{x}$.

Proof. The proof follows on the lines of Schroeder [16] by observing that $G(y, x)>0$ for all $x \in X$ and $y \in Y$.

LEMMA 4. Let $\bar{x}$ be an optimal solution of (NP) with the optimal value $\bar{v}$. Then $\bar{v}$ is the value of the ratio game $J(y, x)$ and $\bar{x}$ is an optimal strategy for Player II and conversely.

Now following exactly as in the linear case, we have the following convex program $(C P)_{v}$ (in place of $(L P)_{v}$ ) for a given $v \in R$ :

$(C P)_{0}: \quad$ Minimize $s$ subject to

$$
\begin{aligned}
f_{l}(x)-v g_{i}(x) \leqslant s, & i=1,2, \ldots, p \\
h_{k}(x) \leqslant 0, & k=1,2, \ldots, r .
\end{aligned}
$$


The dual $(N D)$ of the primal $(N P)$ will depnd upon the dual $D(C P)_{v}$ of the convex program $(C P)_{v}$. Since there could be many ways of writing $D(C P)_{v}$, the dual $(N D)$ will not be unique. By taking the Lagrangian dual of $(C P)_{v}$ and following techniques similar to the linear case, we get $(N D)$ in the form of [3] and [10]. However, if we assume that the functions $f_{i}, g_{i}, h_{k}$ are differentiable and program $(C P)_{v}$ satisfies an appropriate constraint qualification (Craven [2], Mangasarian [13]), then $D(C P)_{v}$ can be taken as the following Wolfe dual:

$D(C P)_{v}: \quad$ Maximize $\sum_{\imath=1}^{p} u_{t}\left[f_{t}(x)-v g_{\imath}(x)\right]+\sum_{k=1}^{r} w_{k} h_{k}(x)$

subject to

$$
\begin{gathered}
\sum_{i=1}^{p} u_{i} \nabla f_{i}(x)-v \sum_{i=1}^{p} u_{i} \nabla g_{i}(x)+\sum_{k=1}^{r} w_{k} \nabla h_{k}(x)=0, \\
u \geqslant 0, w \geqslant 0 \\
u \neq 0 .
\end{gathered}
$$

Now as in the linear case, we define the following problem $(N D)$ as the dual of the primal $(P)$ :

Dual (ND): $\quad$ Maximize $v$ subject to

$$
\begin{gathered}
\sum_{i=1}^{p} u_{i} f_{i}(x)-v \sum_{i=1}^{p} u_{i} g_{i}(x)+\sum_{k=1}^{r} w_{k} h_{k}(x) \geqslant 0, \\
\sum_{i=1}^{p} u_{i} \nabla f_{i}(x)-v \sum_{i=1}^{p} u_{i} \nabla g_{i}(x)+\sum_{k=1}^{r} w_{k} \nabla h_{k}(x)=0 \\
u \geqslant 0, w \geqslant 0 \\
u \neq 0 .
\end{gathered}
$$

Duality results similar to Theorem 1 (Weak Duality) and Theorem 2 (Strong and Converse Duality) hold between $(N P)$ and $(N D)$. The proofs are not given here as they follow exactly on the lines of the linear case. It is simple to observe that in case $f_{t}, g_{t}, h_{k}$ are affine and $x \geqslant 0$ is explicitly present in the set $X$, the dual $(N D)$ reduces to the linear case dual i.e. dual $(D)$.

\section{Algorithmic aspects}

The equivalence between generalized fractional programming and ratio game theory immediately opens various possibilities of developing solution procedures for problems of type $(P)$ and $(N P)$. 
In the linear case, we can simply translate Schroeder's computational procedure for ratio games [16] and get an algorithm for solving $(P)$. This involves solving $(L P)_{v}$ for a sequence of parameters $v$ until a zero value is obtained. The basic tools used in [16] are the Simplex Method and certain linear programming perturbation results which give the variation in the optimal value of $(L P)_{v}$ that results from a perturbation in $v$. A related approach (without proof of convergence) for the linear case was given in [1], and both linear and nonlinear results were given in [5]. For the nonlinear case, we can hope to follow a similar procedure, provided we have certain convex programming perturbation results for $(C P)_{v}$ which also help in demonstrating the convergence of such a procedure. We shall explore these aspects more fully in subsequent work.

\section{Acknowledgment}

One of the authors (S. Chandra) acknowledges the financial support and excellent research facilities provided by the authorities of the University of Melbourne during his term of a visiting research fellowship.

The authors thank two referees for some detailed amendments.

\section{References}

[1] A. Charnes and W. W. Cooper, "Goal programming and multiobjective optimization, Part I," European J. Oper. Res. 1 (1979), 39-54.

[2] B. D. Craven, Mathematical programming and control theory (Chapman and Hall, London, 1978).

[3] J. P. Crouzeix, "A duality framework in quasiconvex programming," in "Generalized concavity in optımization and economics, (eds.) S. Schaible and W. T. Ziemba, (Academic Press, New York, 1981), 207-225.

[4] J. P. Crouzeix, J. A. Ferland and S. Schaible, "Duality in generalized fractional programming", Math. Programming 27 (1983), 342-354.

[5] J. P. Crouzeix, J. A. Ferland and S. Schaible, "An algorithm for generalized fractional programs", J. Oprim. Theory Appl. 47 (1985), 35-49.

[6] W. Dinkelbach, "On nonlinear fractional programming", Management Sct. 13 (1967), 492-498.

[7] K. Fan, "Minimax theorems", Proc. Nat. Acad. Sci. U.S. A. 39 (1953), 42-47.

[8] R. Jagannathan, "On some properties of programming problems in parametric form pertaining to fractional programming", Management Sci. 12 (1966), 609-615.

[9] R. Jagannathan, "Duality for nonlinear fractional programs", Zeits. Oper. Res. 17 (1973), 1-3.

[10] R. Jagannathan and S. Schaible, "Duality in generalized fractional programming via Farkas lemma", J. Optim. Theory Appl. 41 (1983), 417-424.

[11] S. Karlin, Mathematical methods and theory in games, programming and mathemattcal economics (Addison-Wesley, Reading, Mass., 1959). 
[12] L. H. Lommis, "On a theory of von Neumann", Proc. Nat. Acad. Sci. U.S.A. 32 (1946), 213-215.

[13] O. L. Mangasarian, Nonlmear programming (McGraw-Hill, New York, 1969).

[14] S. Schaible, "Bibliography in fractional programming", Zeits. Oper. Res. 26 (1982), 211-241.

[15] S. Schaible, "Fractional programming-invited survey", Zetts. Oper. Res. 27 (1983), 30-54.

[16] R. G. Schroeder, "Linear programming solutions to ratio games, Oper. Res. 18 (1970), 300-305.

[17] L. S. Shapley, "Stochastic games", Proc. Nat. Acad. Sci. U.S. A. 39 (1953), 1095-1100

[18] M. Sion, "On general minimax theorems", Pacific J. Math. 8 (1958), 171-176.

[19] J. von Neumann, "A model of general economic equilibrium", Rev. Econom. Stud. 13 (1945), 1-9. 